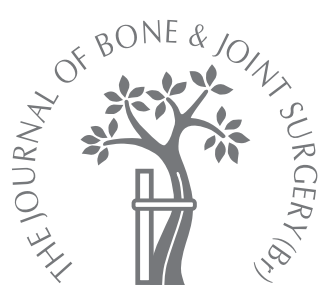
A. Finestone,
C. Milgrom,
D. R. Radeva-Petrova,
E. Rath,
V. Barchilon,
S. Beyth,
S. Jaber,
O. Safran

From Hadassah

University Hospital,

Jerusalem, Israel

A. Finestone, MD,

Orthopaedic Surgeon

Assaf HaRofeh Medical Centre

Zerrifin, Beer Yaakov 70300,

Israel.

C. Milgrom, MD, Professor

D. R. Radeva-Petrova, MPH,

Clinical Research Assistant

- S. Beyth, MD, Orthopaedic

Surgeon

S. Jaber, MD, Orthopaedic

Surgeon

O. Safran, MD, Orthopaedic

Surgeon

Department of Orthopaedics

Hadassah University Hospital,

Ein Kerem, P. O. Box 12000

Jerusalem, Israel.

E. Rath, MD, Orthopaedic Surgeon

Soroka Hospital, Ben Gurion

University Medical School,

POB151 Beersheva 84141,

Israel.

- V. Barchilon, MD

Orthopaedic Surgeon

Meir Hospital, Sapir Medical

Centre, Tchernitiovsky 59, Kfar

Sava 44281, Israel.

Correspondence should be sent to Professor C. Milgrom; e-mail: charlesm@ekmd.huji.ac.il

(c)2009 British Editorial Society of Bone and Joint Surgery doi:10.1302/0301-620X.91B7. $22263 \$ 2.00$

$J$ Bone Joint Surg $[\mathrm{Br}]$ 2009;91-B:918-21.

Received 5 January 2009;

Accepted after revision 27

March 2009

\title{
Bracing in external rotation for traumatic anterior dislocation of the shoulder
}

\begin{abstract}
We undertook a prospective study in $\mathbf{5 1}$ male patients aged between $\mathbf{1 7}$ and 27 years to ascertain whether immobilisation after primary traumatic anterior dislocation of the shoulder in external rotation was more effective than immobilisation in internal rotation in preventing recurrent dislocation in a physically active population.

Of the 51 patients, 24 were randomised to be treated by a traditional brace in internal rotation and 27 were immobilised in external rotation of $15^{\circ}$ to $20^{\circ}$. After immobilisation, the patients undertook a standard regime of physiotherapy and were then assessed clinically for evidence of instability. When reviewed at a mean of 33.4 months (24 to 48 ) ten from the external rotation group (37\%) and ten from the internal rotation group (41.7\%) had sustained a futher dislocation. There was no statistically significant difference $(p=0.74)$ between the groups.

Our findings show that external rotation bracing may not be as effective as previously reported in preventing recurrent anterior dislocation of the shoulder.
\end{abstract}

The aim of treatment for primary anterior traumatic dislocation of the shoulder is to prevent subsequent recurrent dislocation. The traditional method of management is by immobilisation in internal rotation. Although this method has been shown to be ineffective in lowering the incidence of recurrent dislocation, ${ }^{1,2}$ it is widely used. When followed up at two years the rate of recurrence after a primary dislocation was found to be the same for shoulders immobilised for three to four weeks in internal rotation as in those which were left free. ${ }^{1}$

As the shoulder dislocates anteriorly, the movement of the head of the humerus can cause either an anterior labral tear and/or interstitial capsular stretching and tearing. In younger patients there is usually an anterior labral tear while in older patients stretching and tearing of the capsule ${ }^{3}$ commonly occurs. If the torn labrum heals in place after the first dislocation, the risk of recurrence is expected to decrease. ${ }^{4}$

Itoi et $\mathrm{al}^{4}$ used MRI to assess the position of the labral tear after dislocation of the shoulder and concluded that displacement of the labrum from the anatomical position is less when the shoulder is in external rotation and greater when in internal rotation. They therefore proposed that bracing the shoulder in external rotation after a primary dislocation might lower the incidence of recurrence.
In a subsequent randomised prospective study, ${ }^{5}$ they recorded a lower rate of recurrence of dislocation in patients treated by external rotation bracing compared with those managed in internal rotation.

Our aim therefore was to ascertain if managing a primary traumatic dislocation of the shoulder by immobilisation in external rotation was more effective than in internal rotation in lowering the incidence of recurrent dislocations in a young, physically active population.

\section{Patients and Methods}

From January 2004, male patients between the age of 17 and 27 years who had sustained a traumatic anterior dislocation of the shoulder were randomised to be treated for four weeks using either a traditional internal rotation brace (Fig. 1) or a new device which immobilised the shoulder at $15^{\circ}$ to $20^{\circ}$ of external rotation (Fig. 2). Informed consent was obtained from all the participants and the study was approved by the Institutional Review Board. Patients were excluded if they had sustained the injury in a motor-vehicle accident or had a concurrent fracture of the greater tuberosity. There were 51 patients in the study with a mean age of 20.3 years (17 to 27). Of the 51 patients 40 were soldiers. 


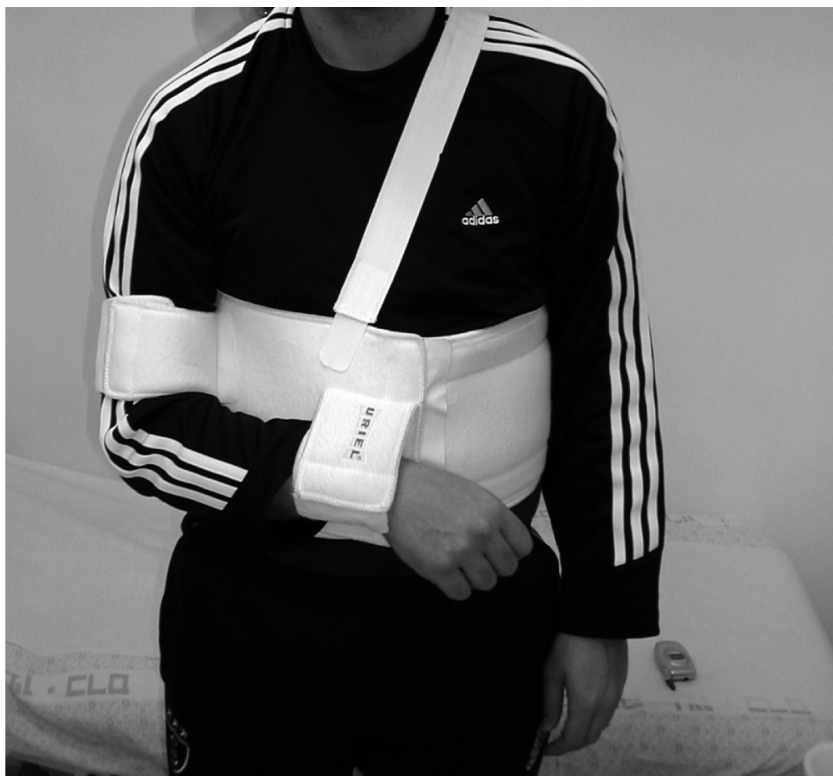

Fig. 1

Photograph showing the traditional method of treatment of a primary traumatic anterior dislocation of the shoulder by immobilisation in internal rotation.

The patients were allowed to remove the brace to shower and to change their clothes but otherwise wore it continuously. After immobilisation for four weeks they began a standard regime of physiotherapy. They were reviewed at two, six and 12 weeks, six months and at one, two, three and four years after the initial injury. The follow-up at one year and later was done by a single observer (CM). Patients who were considered to have clinically stable shoulders on the basis of a negative supine apprehension test returned to full activity after three months. Those who sustained re-dislocations were evaluated by either CT or MRI arthrography.

Statistical analysis. On the basis of a previous study by Itoi et $\mathrm{al}^{5}$ we considered that patients in the age group in our study, if immobilised in internal rotation, would have a $50 \%$ chance of recurrent dislocation at a minimum follow-up of two years. It was assumed that immobilisation in external rotation would reduce this to $10 \%$. We calculated that if $55 \%$ of the subjects were immobilised in external rotation and $45 \%$ in internal rotation, a sample size of 22 subjects in the internal rotation group and 26 in the external rotation group would provide $80 \%$ power $(\alpha=0.05)$ to yield a statistically significant result.

The Pearson correlation coefficient and the chi-squared test were used to assess whether there was a statistically significant difference between the rate of recurrent dislocation in the two groups. A p-value of $<0.05$ was considered statistically significant.

\section{Results}

Closed reduction of the initial dislocation was carried out in the emergency department using the Milch $^{6}$ technique in

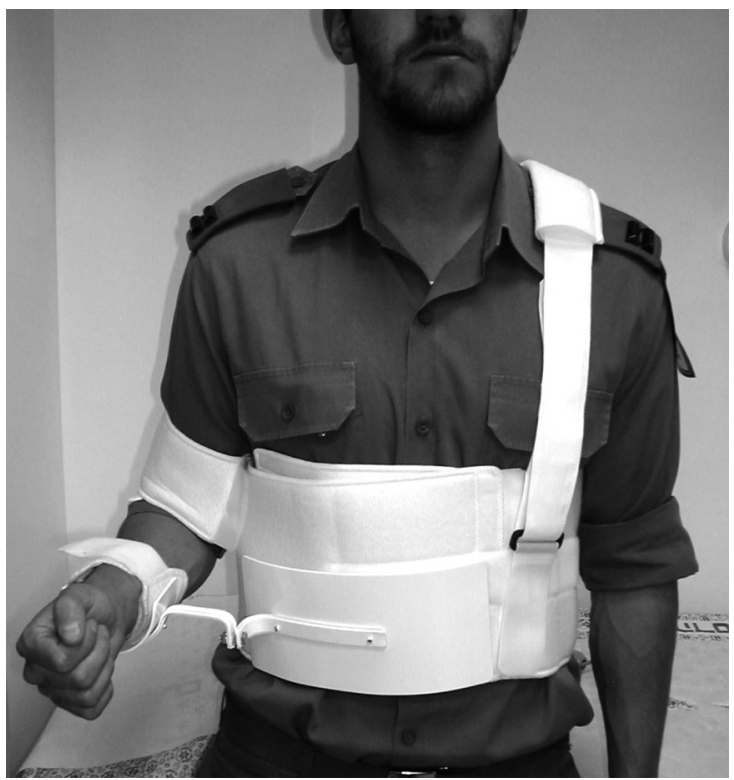

Fig. 2

Photograph showing immobilisation in external rotation of $15^{\circ}$ to $20^{\circ}$.

41 patients $(80 \%)$ and by the Hippocratic technique in the remainder. The external rotation brace was used in 27 patients (Fig. 2), all but one of whom complied fully with the treatment. One patient removed the splint two days early. This was not considered to be a significant deviation from the treatment regime and he was included in the statistical analysis. At a mean follow-up of 35.8 months (24 to 48), ten patients $(37.0 \%)$ had sustained a further dislocation (Table I) at a mean of 13.8 months (4 to 43 ) after the first injury.

An internal rotation brace (Fig. 1) was used in 24 patients, all of whom complied with the treatment regime. At a mean follow-up of 30.8 months (24 to 47 ), ten $(41.7 \%$ ) had sustained a further dislocation (Table I). The new dislocations had occurred at a mean of 12.4 months (4 to 36) after the first injury. There was no statistical difference $(\mathrm{p}=0.74)$ in the rates of instability of the two groups.

Of the 51 patients in the study ten $(19.6 \%)$ sustained neurapraxia of the axillary nerve which resolved within ten weeks in all cases. An axillary rash developed in two patients immobilised in internal rotation. There were no fractures of the glenoid rim. Of the 20 subjects with recurrent dislocation, six had undergone surgery when last reviewed. All had a Bankart lesion. The CT or MRI arthrograms of 13 of the remaining 14 patients who had sustained a re-dislocation showed a Bankart lesion, one of which was bony.

\section{Discussion}

After a primary anterior dislocation of the shoulder treatment by bracing for three to four weeks allows optimal healing of the soft-tissue damage. Watson-Jones ${ }^{7}$ advised immobilisation in internal rotation for a period of at least 
Table I. Details of recurrent dislocations of the shoulder

\begin{tabular}{llll}
\hline & External rotation & Internal rotation & Total \\
\hline No recurrent dislocation & 17 & 14 & 31 \\
Recurrent dislocation & 10 & 10 & 20 \\
Total & 27 & 24 & 51 \\
\hline
\end{tabular}

three weeks. Until recently, the conventional management had been by bracing the shoulder in internal rotation. ${ }^{8}$ However, in a prospective multicentre study to evaluate the results of conservative treatment of primary anterior dislocation of the shoulder, Hovelius et $\mathrm{al}^{1}$ found that bracing in internal rotation did not prevent recurrence after a primary dislocation. In their study, 112 patients were immobilised in internal rotation for three to four weeks, while 104 began to use the dislocated shoulder as early and as freely as possible. When followed up at two years the groups showed an equal rate of recurrent dislocation. The patients had both traumatic and non-traumatic dislocations.

Itoi et $\mathrm{al}^{9}$ presented a different concept for the treatment of primary traumatic anterior dislocation of the shoulder. They carried out experiments on cadavers and then used MRI to assess the optimal position of immobilisation to reduce the anterior labral tear which is characteristic of a traumatic dislocation of the shoulder. ${ }^{4}$ A total of 19 shoulders in patients who had sustained either a primary traumatic or a recurrent anterior dislocation were evaluated. All had MRI to examine coaptation of a Bankart lesion with the arm held at the side of the trunk and positioned first in internal rotation (mean, $29^{\circ}$ ) and then in external rotation (mean, $\left.35^{\circ}\right){ }^{4}$ The separation and displacement of the anteroinferior portion of the labrum from the glenoid rim were measured on the axial images, and coaptation of the anterior part of the capsule to the neck of the glenoid was assessed by measurement of the detached area, the opening angle and the detached length. There was less separation $(\mathrm{p}=0.0047)$ and displacement $(\mathrm{p}=0.0017)$ of the labrum when the arm was in external rotation than when in internal rotation. The detached area and the opening angle of the anteroinferior portion of the capsule were both significantly smaller and the detached length was significantly shorter with the arm in external rotation. They concluded that immobilisation of the arm in external rotation approximated the Bankart lesion to the neck of the glenoid better than in the conventional position of internal rotation.

In the classical Bankart lesion, there is avulsion of the anteroinferior labrum from the underlying glenoid fossa, with displacement and disruption of the periosteum of the scapula. In 1906, Perthes ${ }^{10}$ first described a lesion of the labrum in which there was an incomplete avulsion of its anteroinferior aspect with medial stripping of the periosteum which remained intact. This lesion is now considered to be a variant of the Bankart lesion. Seybold et al, ${ }^{11}$ used MRI to determine if there was a difference in the effectiveness of positioning in external rotation in reducing the labrum between classical Bankart lesions and Perthes lesions. They found that external rotation was more effective in Perthes lesions, and was greatest when there was little or no deformation of the anterior labroligamentous structures. They also found that the incidence of Perthes and classical Bankart lesions was equal in their patients with primary traumatic dislocations.

After their MRI study, ${ }^{4}$ Itoi et $\mathrm{al}^{5}$ carried out a preliminary prospective clinical study. They assigned 40 patients with primary dislocations either to conventional immobilisation in internal rotation or to immobilisation in $10^{\circ}$ of external rotation. Initially, they attempted to immobilise the shoulder in $30^{\circ}$ of external rotation, but this was not well tolerated by the patients. Therefore they decreased the angle of external rotation "arbitrarily down to $10^{\circ}$ ". The rate of recurrence was $30 \%$ in the internal rotation group and zero in those in external rotation at a mean follow-up of 15.5 months. In subjects under the age of 30 years there was a rate of recurrence of $45 \%$ in the internal rotation and of zero in the external rotation group.

In a more recent randomised, controlled trial carried out by Itoi et al, ${ }^{12} 136$ male and 62 female patients with a primary anterior dislocation of the shoulder were randomly assigned to be treated by immobilisation in either external rotation at $10^{\circ}$ or in internal rotation. Their mean age was 37 years (12 to 90$)$. Of the 198 patients 98 (49.5\%) did not comply with the treatment protocol for the recommended minimum period of three weeks. Both the per-protocol and intention-to-treat analyses showed that the recurrence rate in the external rotation group was significantly lower than that in those in internal rotation.

In our study, 20 of 51 patients $(39.2 \%)$ sustained a recurrent dislocation. Although the follow-up period was short our findings agree with those of Hovelius et al, ${ }^{13}$ in suggesting that the rate of recurrence after a primary dislocation in a young population is lower than previously reported. Our study found no statistical difference in the rate of recurrence between those immobilised in external or internal rotation. Our study may have been underpowered but our findings strongly support the hypothesis that there was no difference between the treatment groups. Our patients were young, physically active men. Their compliance with the treatment was excellent although they reported that initially it was not easy to adjust to the external rotation brace. The compliance was undoubtedly improved by the fact that most were soldiers who had a high degree of self-discipline and were treated within a military framework. After treatment for dislocation they 
resumed vigorous physical activity which tested the stability of the shoulder to the utmost. Our patients therefore were very dissimilar to those in the study of Itoi et $\mathrm{al}^{12}$ who were of mixed gender and aged from 12 to 90 years (mean 37). Young age and male gender have been found to be important determinants of the risk of recurrent instability. ${ }^{14}$ In our study 19 of 20 CT or MRI arthrograms of re-dislocations showed a Bankart lesion, confirming the clinical classification of traumatic dislocation. The study of Itoi et al ${ }^{12}$ lacks such data.

The probability of the presence of a Bankart lesion in an older patient with a primary dislocation is low, ${ }^{3}$ as is the risk of recurrence. ${ }^{15,16}$ In a primary dislocation there is characteristically a failure of one or more of the anterior stabilising structures of the shoulder. Under the age of 30 years, this is usually a tear of the anterior labrum, with or without a fracture fragment from the glenoid. After the age of 30 years labral tears are less common ${ }^{17}$ and rupture of the capsule is more likely. The shoulders of our patients were immobilised at $15^{\circ}$ to $20^{\circ}$ of external rotation which is more than $10^{\circ}$ used by Itoi et al. ${ }^{5}$ Based on the MRI study of the position of the labrum by Itoi et $\mathrm{al}^{4}{ }^{4}$ the greater external rotation which we used would have been expected to increase the effectiveness of the brace treatment.

Our study suggests that external rotation bracing may not be as effective as previously described in preventing recurrent dislocations of the shoulder.

This study has been supported by a research grant of the IDF Medical Corporation and Israeli Ministry of Defence.

No benefits in any form have been received or will be received from a com mercial party related directly or indirectly to the subject of this article.

\section{References}

1. Hovelius L, Eriksson K, Fredin H, et al. Recurrences after initial dislocation of the shoulder: results of a prospective study of treatment. J Bone Joint Surg [Am]1983;65A:343-9.

2. Henry JH, Genung JA. Natural history of glenohumeral dislocation: revisited. Am J Sports Med 1982;10:135-7.

3. Wen DY. Current concepts in the treatment of anterior shoulder dislocations. Am J Emerg Med 1999;17:401-7.

4. Itoi E, Sashi R, Minagawa $\mathbf{H}$, et al. Position of immobilization after dislocation of the glenohumeral joint: a study with use of magnetic resonance imaging. J Bone Joint Surg [Am] 2001;83-A:661-7.

5. Itoi E, Hatakeyama Y, Kido T, et al. A new method of immobilization after traumatic anterior dislocation of the shoulder: a preliminary study. J Shoulder Elbow 2003:12:413-51.

6. Milch H. Treatment of dislocation of the shoulder. Surgery 1938;3:732-40.

7. Watson-Jones R. Fractures and joint injuries. Vol. 2. Fourth ed. Baltimore: Williams \& Wilkins Company, 1955:477

8. Rowe CR. Prognosis in dislocations of the shoulder. J Bone Joint Surg [Am]1956;38A:957-77.

9. Itoi E, Hatakeyama $\mathbf{Y}$, Urayama $\mathbf{M}$, et al. Position of immobilization after dislocation of the shoulder: a cadaveric study. J Bone Joint Surg [Am] 1999;81-A:385-90.

10. Perthes G. Über operationen bei habitueller schulterluxation. Dtsch Z Chir 1906;85:199-227.

11. Seybold D, Schliemann B, Heyer CM, Muhr G, Gekle C. Which labral lesion can be best reduced with external rotation of the shoulder after a first-time traumatic anterior shoulder dislocation? Arch Orthop Trauma Surg 2008;29:318-24.

12. Itoi E, Hatakeyama $Y$, Sato $T$, et al. Immobilization in external rotation after shoulder dislocation reduces the risk of recurrence: a randomized controlled trial. J Bone Joint Surg [Am] 2007;89-A:2124-31.

13. Hovelius L, Olofsson A, Sandstrom B, et al. Nonoperative treatment of primary anterior shoulder dislocation in patients forty years of age and younger: a prospective twenty-five year follow-up. J Bone Joint Surg [Am] 2008;90-A:945-52.

14. Robinson CM, Howes J, Murdoch H, Will E, Graham C. Functional outcome and risk of recurrent instability after primary traumatic anterior shoulder dislocation in young patients. J Bone Joint Surg [Am] 2006;88-A:2326-36.

15. Kazar B, Relovszky E. Prognosis of primary dislocation of the shoulder. Acta Orthop Scand 1969;40:216-24.

16. Kiviluoto $\mathbf{0}$, Pasila $\mathbf{M}$, Jaroma $\mathbf{H}$, Sundholm $\mathbf{A}$. Immobilization after primary dislocation of the shoulder. Acta Orthop Scand 1980;51:915-19.

17. Reeves B. Experiments on the tensile strength of the anterior capsular structures of the shoulder in man. J Bone Joint Surg [Br] 1968;50-B:858-65, 